\title{
El embarazo en mujeres con lupus eritematoso sistémico: una revisión integrativa
}

\author{
Patricia Mesa-Abad ${ }^{1}$, Lucía Tovar-Muñoz ${ }^{1}$, Irene Serrano-Navarro' ${ }^{1}$, Pedro Ventura-Puertos ${ }^{3}$, José Berlango- \\ Jiménez ${ }^{1,2}$ \\ ${ }^{1}$ Departamento de Enfermería. Facultad de Medicina y Enfermería. Universidad de Córdoba. Córdoba. España \\ ${ }^{2}$ Servicio de Nefrología. Hospital Universitario Reina Sofía de Córdoba. Córdoba. España \\ ${ }^{3}$ Instituto Maimónides de Investigación Biomédica de Córdoba. Córdoba. España
}

Como citar este artículo:

Mesa-Abad P, Tovar-Muñoz L, Serrano-Navarro I, Ventura-Puertos P, Berlango-Jiménez J. El embarazo en mujeres con lupus eritematoso sistémico: una revisión integrativa. Enferm Nefrol. 2020 Ene-Mar;23(1):11-21

\section{Resumen}

Introducción: En la actualidad, se conoce que el lupus eritematoso sistémico no afecta a la fertilidad; sin embargo, el embarazo en mujeres que lo padecen se asocia a riesgos, tanto maternos como fetales.

Objetivo: Determinar las variables que intervienen en la preparación y control de la gestación en mujeres con lupus eritematoso sistémico, así como las medidas de enfermería más importantes.

Material y Método: Se ha realizado una revisión integrativa mediante una búsqueda en las bases de datos Proquest, Pubmed y Web of Science. Se incluyeron artículos científicos originales, observacionales y de revisión, en inglés y español, excluyendo aquellos artículos anteriores a 2009 o que no estuviesen en texto completo.

Resultados: Se han incluido 15 artículos publicados entre el año 2009 y 2019. El embarazo en pacientes con lupus eritematoso sistémico es complejo, ya que necesita de un control exhaustivo y continuo para conseguir buenos resultados. Un cambio de tratamiento, test de laboratorio cada cierto tiempo y la vigilancia de un equipo multidisciplinar, harán que la enfermedad se encuentre controlada y los riesgos de complicaciones maternas y fetales disminuyan.

\section{Correspondencia:}

Patricia Mesa Abad

E-mail: pati_1297@hotmail.com
Conclusiones: En la actualidad, la mayoría de las mujeres con lupus eritematoso sistémico pueden conseguir el objetivo de la maternidad, siempre y cuando la enfermedad se encuentre controlada al menos 6 meses antes de la concepción y durante todo el embarazo. Las variables más determinantes en el embarazo de la mujer con lupus eritematoso sistémico son la fertilidad, las complicaciones o el tratamiento, que influyen de forma directa sobre el embarazo.

PALABRAS CLAVE: lupus eritematoso sistémico; fertilidad; complicaciones embarazo; control embarazo; intervenciones de enfermería.

\section{Pregnancy in women with systemic lupus erythematosus: an integrative review}

\section{Abstract}

Introduction: At present, it is known that systemic lupus erythematosus does not affect fertility; however, pregnancy in these women is associated with risks, both maternal and fetal.

Objective: To determine the variables involved in the preparation and control of pregnancy in women with systemic lupus erythematosus, as well as the most important nursing actions.

Material and Method: An integrative review has been carried out through a search in the ProQuest, PubMed 
and Web of Science databases. Original, observational and review scientific articles were included, in English and Spanish, excluding those articles published before 2009 or that were not in full text in the databases.

Results: 15 articles published between 2009 and 2019 have been included. Pregnancy in patients with systemic lupus erythematosus is complex. Comprehensive and continuous control is required to achieve good results. A change of treatment, laboratory tests from time to time and the monitoring of a multidisciplinary team will control the disease and will reduce the risk of maternal and fetal complications.

Conclusions: Currently, most women with systemic lupus erythematosus can achieve the goal of motherhood, if the disease is controlled at least 6 months before conception and throughout pregnancy. The most determinant variables in pregnancy of women with systemic lupus erythematosus are fertility, complications or treatment, which directly influence pregnancy.

KEYWORDS: systemic lupus erythematosus; fertility; pregnancy complications; pregnancy control; nursing interventions.

\section{Introducción}

El Lupus Eritematoso Sistémico (LES) se conoce desde hace quinientos años y las primeras publicaciones fueron en los siglos XV y XVI donde se denominó "lupus" por las características ulceraciones que aparecen en el rostro y las cuales se relacionaron con la mordedura de 1 lobo $^{1}$.

EI LES una enfermedad de origen autoinmune en la que el organismo crea autoanticuerpos que dañan células, tejidos y órganos sanos. Se da con más frecuencia en el género femenino en edad fértil $y$, al tratarse de una enfermedad reumática crónica y grave, un posible embarazo debe ser planificado y guiado por un equipo multidisciplinar para lograr un fin exitoso y con un menor riesgo, además de encontrarse controlada en el momento de concebir ${ }^{1-5}$.

Estudios epidemiológicos han demostrado un aumento de la incidencia del LES en los últimos 30 años, que puede atribuirse a un diagnóstico más temprano. Lo sufren 1 de cada 1000 mujeres, las cuales tienen 2-4 veces más probabilidades de complicaciones en el embarazo que aquellas que no padecen la enfermedad ${ }^{6,7}$.
En la actualidad, se conoce que el LES no afecta a la fertilidad, por lo que el embarazo en estas mujeres no resulta complicado. Sin embargo, están expuestas a mayores riesgos, tanto maternos como fetales, los cuales pueden disminuir si se elaboran estrategias de tratamiento específicas e individualizadas ${ }^{4,6-9}$. Este aspecto es esencial, puesto que se deberá sustituir toda terapia teratógena por otras alternativas que no causen daño al feto y que, a su vez, controlen los brotes maternos $3,6,9-12$.

Algunos de los medicamentos que tratan el lupus son: corticosteroides, hidroxicloroquina, inmunosupresores o un tratamiento biológico como el belimumab. Esto es importante de cara a un posible embarazo ya que las pacientes podrán y deberán continuar con su terapia de Hidroxicloroquina que protegerá de posibles exacerbaciones de la enfermedad y trombosis, así como se ha comprobado que se consigue una mayor tasa de nacimientos vivos en pacientes que además sufren síndrome antifosfolípido; pero no con inmunosupresores por su teratogenia. Éstos últimos son tóxicos para el feto pudiendo provocar bajo peso al nacer, prematuridad $u$ otras complicaciones $3,7,8,10-12$.

Los riesgos de la gestante dependerán de la actividad de la enfermedad antes de la concepción y a lo largo de todo el embarazo, la afectación de algunos órganos y la presencia de autoanticuerpos. Los riesgos fetales van a depender de la actividad de la enfermedad de la madre, la presencia de autoanticuerpos y el tratamiento que esta esté tomando durante el embarazo $0^{4,6,12}$.

Aproximadamente en un $40-50 \%$ de los embarazos en mujeres con LES existe actividad lúpica, y aparece con mayor frecuencia en mujeres con enfermedad activa en los últimos 6-12 meses. Por ello, se debe evaluar de forma global a la paciente y asegurar que se trata de un momento idóneo para la concepción. Sin embargo, esta actividad suele empeorar en el postparto, al aumentar los niveles de estrógenos, prolactina y citocinas $\mathrm{s}^{4,6,8,9,12}$.

Por tanto, y dados los riesgos potenciales en el embarazo de las mujeres afectadas de LES, es fundamental el papel de los equipos multidisciplinares, los cuales planificarán el embarazo de estas mujeres durante un periodo sin actividad de la enfermedad o en remisión, modificarán el tratamiento de base por fármacos no teratogénicos y tratarán los síntomas que vayan apareciendo y que pongan en riesgo la vida de la madre 0 del feto $0^{6,9}$. 
Por todo ello, el objetivo de esta revisión ha sido determinar las variables que intervienen en la preparación y control de la gestación en mujeres con LES, así como las medidas de enfermería más importantes en el cuidado de estas mujeres.

\section{Material y Método}

\section{Diseño}

El diseño empleado es el de un estudio de revisión integrativa.

\section{Estrategia de búsqueda}

El desarrollo de la revisión integrativa conlleva distintas fases. De esta manera para la elaboración de ésta se han llevado a cabo las siguientes:

- Identificación del objetivo de la revisión: Revisar la producción científica existente sobre la gestación en pacientes con lupus eritematoso sistémico.

- Determinación de los criterios de búsqueda: La búsqueda de bibliografía se realizó en diversas bases de datos con el objetivo de recopilar la mayor producción posible disponible con respecto al tema a tratar. En un principio se eligió un periodo de 5 años mediante la realización de una revisión sistemática, pero ante la falta de bibliografía se decidió ampliar la revisión a los últimos 10 años (entre 2009 y 2019) y elaborar una revisión integrativa. Las bases de datos a las que se accedió fueron: Proquest, Pubmed y Web of Science. EI periodo de consulta tuvo lugar desde marzo de 2019 hasta mayo del mismo año.

El método de búsqueda consistió en utilizar como palabras clave: lupus eritematoso sistémico (systemic lupus erythematosus), prevalencia (prevalence), fertilidad (fertility), complicaciones embarazo (pregnancy complications), tratamiento (treatment), control embarazo (pregnancy control), factores de riesgo (risk factors), intervenciones de enfermería (nursing interventions), unidos dichos términos por el conector booleano "AND". En cada base de datos se llevó a cabo estrategias de búsqueda diferentes, realizando distintas pruebas de asociación entre los descriptores anteriormente citados, teniendo en cuenta que cada uno de los descriptores empleados estuvieran presentes en el resumen, título del artículo y, si la base de datos lo presenta, en palabras clave, y de acuerdo a los siguientes criterios de inclusión: estudios observacionales y de revisión bibliográfica, artículos en inglés y en español y artículos en los cuales se tratara la gestación en mujeres con lupus eritematoso sistémico. Los criterios de exclusión fueron: artículos anteriores a 2009 y artículos sin texto completo disponible.

En la Tabla 1, se representan los artículos encontrados en la primera búsqueda y los seleccionados.

Tabla 1. Tabla de resultados de la búsqueda.

\begin{tabular}{|l|c|c|c|}
\hline Bases de datos & Proquest & Pubmed & Web of Science \\
\hline $\begin{array}{l}\text { Total de publicaciones } \\
\text { localizadas }\end{array}$ & 98 & 19 & 103 \\
\hline $\begin{array}{l}\text { Total de publicaciones } \\
\text { seleccionadas }\end{array}$ & 10 & 1 & 4 \\
\hline
\end{tabular}

- Selección de los documentos incluidos en el estudio: Tras la lectura de los títulos y resúmenes de todos los artículos, se fueron descartando aquellos que no cumplían con los criterios de inclusión y exclusión. Posteriormente se realizó la lectura completa de los trabajos seleccionados y se realizó un nuevo descarte de aquellos que no versaban sobre el tema a estudiar. De un total de 220 artículos preseleccionados, se excluyeron 128 durante la revisión de títulos y resúmenes. Otros 56 fueron eliminados tras la revisión del texto completo. Finalmente, se seleccionaron 15 artículos que cumplían con los criterios de inclusión/exclusión previamente establecidos, tal como se refleja en la Figura 1.

\section{- Determinación de la información a obtener de los} artículos: tras la lectura de los documentos a texto completo, se decidió agrupar la información en las siguientes categorías: definición de lupus eritematoso sistémico, prevalencia actual del mismo en cuanto a gestación, relación entre lupus y fertilidad, complicaciones asociadas al embarazo en el lupus, tratamiento durante el periodo de gestación e intervenciones de enfermería relativas al control del embarazo en el lupus.

- Análisis del conocimiento: En esta fase se procedió a la interpretación de la información recopilada.

- Síntesis de resultados: Se llevó a cabo la integración de las conclusiones obtenidas. 




Figura 1. Diagrama de flujo de la selección de artículos. del LES en España es limitada, pero estudios recientes en Europa y Estados Unidos sugieren un aumento de la incidencia y la mejora de las tasas de supervivencia en esta enfermedad $^{14}$.

Debido a que la edad en la que suele aparecer esta enfermedad corresponde al período fértil de la mujer, existen numerosas preocupaciones acerca de un posible embarazo ${ }^{14,18}$. Estudios confirman que la mortalidad materna en el LES es hasta 20 veces mayor que la tasa general $7,9,16,18,20,24$. Esto se debe a numerosas complicaciones y brotes de la propia enfermedad que aparecen durante la gestación por cambios fisiológicos y hormonales, lo que conlleva a una superproducción de estrógenos, progesterona y citoquinas $8,13,17,24$. La mayor actividad del LES suele ocurrir en el primer y segundo trimestre del embarazo $(90,9 \%$ de los casos) y tras el parto $8,9,13,15,17$. No obstante, hoy en día se ha reducido un $43 \%$ las complicaciones fetales con respecto a los últimos 40 años ${ }^{16,22}$.

En 2014 se publicó un estudio donde en un período de 5 años sólo se documentaron 15 embarazos en pacientes con lupus ${ }^{23}$. La edad avanzada al concebir, las probabilidades de complicaciones o muerte materna, los miedos y la falta de información, hacen que aumente la incidencia de ansiedad y depresión en este tipo de pacientes $(60 \%)$, convirtiéndose esto en un número reducido de descendientes $15,18,20,23,24$.

En la actualidad, las mujeres con LES siguen teniendo numerosas complicaciones en el embarazo (de 2 a 20 veces más que las que no lo padecen) 9,17,20,24. Por ello, un buen control sistemático, exhaustivo y multidisciplinario basado en recomendaciones por expertos debido a la insuficiente evidencia científica $7,8,9,15,17,18,21,24$, puede lograr un embarazo exitoso en la mayoría de estas mujeres, aunque exista mayor o menor riesgo dependiendo de la actividad de la enfermedad en el momento de concebir y durante la gestación $8,9,16,18,20,24$.

Otros autores afirman que una mujer con LES puede tener un embarazo normal, pero se encuentra expuesta a un mayor número de complicaciones ya que intervienen las del propio embarazo y las que puedan surgir por actividad de la enfermedad ${ }^{25}$. 
Tabla 2. Artículos del estudio.

\begin{tabular}{|c|c|c|c|}
\hline $\begin{array}{l}\text { Autor, año y } \\
\text { lugar }\end{array}$ & $\begin{array}{l}\text { Diseño del } \\
\text { estudio }\end{array}$ & Muestra & Principales conclusiones \\
\hline $\begin{array}{l}\text { Tower C et al. } \\
2011, \text { UK }^{7}\end{array}$ & Editorial. & $\begin{array}{l}\text { Número no } \\
\text { definido de } \\
\text { muestra. }\end{array}$ & $\begin{array}{l}\text { - Efectos adversos del embarazo: preeclampsia, parto prematuro y crecimiento } \\
\text { intrauterino retardado. } \\
\text { - Medicamentos seguros en el embarazo: corticoides y azatioprina. Micofenolato } \\
\text { de Mofetilo y AINEs: teratogénicos. } \\
\text { - El aumento de células TREG (células T reguladoras) ayuda al correcto } \\
\text { desarrollo de la placenta. }\end{array}$ \\
\hline $\begin{array}{l}\text { Ching Soh M } \\
\text { et al. 2014, } \\
\text { Gran Bretaña }\end{array}$ & $\begin{array}{l}\text { Revisión } \\
\text { bibliográfica. }\end{array}$ & $\begin{array}{l}\text { Número no } \\
\text { definido de } \\
\text { muestra. }\end{array}$ & $\begin{array}{l}\text { - Los embarazos en el LES deben ser planificados ya que los brotes son más } \\
\text { comunes en aquellas mujeres con enfermedad activa en la concepción. } \\
\text { - Mujeres con LES tienen alto riesgo de: preeclampsia (14-23\%), eclampsia, } \\
\text { parto prematuro (20-31\%) o crecimiento fetal retardado (5-23\%). } \\
\text { - La fertilidad de mujeres con enfermedades reumáticas no se ve afectada. } \\
\text { - Para tener embarazo exitoso: buen control, enfermedad inactiva durante al } \\
\text { menos } 6 \text { meses antes de la concepción, uso continuado de Hidroxicloroquina, } \\
\text { tratamiento rápido para los brotes y seguimiento cuidadoso de anticuerpos. }\end{array}$ \\
\hline $\begin{array}{l}\text { Singh AG et al. } \\
2015, \text { USA }^{9}\end{array}$ & $\begin{array}{l}\text { Revisión } \\
\text { bibliográfica. }\end{array}$ & $\begin{array}{l}\text { Número no } \\
\text { definido de } \\
\text { muestra. }\end{array}$ & $\begin{array}{l}\text { - Factores de riesgo para el embarazo: nefritis activa o hipertensión. } \\
\text { - Complicaciones: parto pretérmino ( } 21 \% \text { ), preeclampsia }(22,5 \%) \text { y eclampsia } \\
(0,5 \%) \text {. El } 20 \% \text { terminan en abortos espontáneos o mortinatalidad. El 1-2\% } \\
\text { de los bebés de madres con autoanticuerpos pueden desarrollar lupus neonatal. } \\
\text { - Supresión de medicamentos teratogénicos } 3 \text { meses antes de concebir. } \\
\text { No suspender Hidroxicloroquina. No está clara la teratogenicidad de un } \\
\text { tratamiento biológico. } \\
\text { - Control: analíticas semanales (anticuerpos, orina, función renal, etc.) Ecografía } \\
\text { en primer trimestre y mensual a partir de semana } 16 \text {. Medición de la velocidad } \\
\text { Doppler de la arteria umbilical semanalmente a partir del tercer trimestre. }\end{array}$ \\
\hline $\begin{array}{l}\text { Zhang C et al. } \\
2017, \text { China }^{13}\end{array}$ & $\begin{array}{l}\text { Estudio } \\
\text { observacional, } \\
\text { longitudinal } \\
\text { retrospectivo. }\end{array}$ & $\begin{array}{l}97 \text { embaraza- } \\
\text { das ( } 22 \text { de ellas } \\
\text { diagnosticadas } \\
\text { con LES duran- } \\
\text { te la gestación } \\
\text { o puerperio). }\end{array}$ & $\begin{array}{l}\text { - Se detectaron las mismas complicaciones fetales tanto en pacientes de nuevo } \\
\text { inicio de la enfermedad como aquellas que la tenían activa antes y durante el } \\
\text { embarazo. Sin embargo, las complicaciones maternas fueron mayores en las de } \\
\text { nuevo diagnóstico. } \\
\text { - La mayor actividad del LES suele ocurrir en el primer y segundo trimestre de } \\
\text { embarazo }(90,9 \%) \text { Por ello, debe hacerse hincapié en el mayor control durante } \\
\text { los primeros } 6 \text { meses. }\end{array}$ \\
\hline $\begin{array}{l}\text { Alonso MD } \\
\text { et al. 2011, } \\
\text { España }^{14}\end{array}$ & $\begin{array}{l}\text { Estudio } \\
\text { observacional, } \\
\text { longitudinal } \\
\text { prospectivo. }\end{array}$ & $\begin{array}{l}\text { Pacientes diag- } \\
\text { nosticadas con } \\
\text { LES del Hospi- } \\
\text { tal Xeral-Calde } \\
\text { de Lugo durante } \\
\text { el período } \\
\text { 1987-2006. }\end{array}$ & $\begin{array}{l}\text { - Estudios recientes en Europa y Estados Unidos sugieren un aumento en la } \\
\text { incidencia y la mejora de las tasas de supervivencia en el LES. } \\
\text { - La información relativa a la epidemiología del LES en España es limitada. } \\
\text { - El } 90 \% \text { de los pacientes diagnosticados con LES son mujeres, de entre } 19 \text { y } 46 \\
\text { años de edad. }\end{array}$ \\
\hline $\begin{array}{l}\text { Andreoli L } \\
\text { et al. } 2017 \text {, } \\
\text { Italia }^{15}\end{array}$ & $\begin{array}{l}\text { Revisión } \\
\text { bibliográfica. }\end{array}$ & $\begin{array}{l}\text { Número no defi- } \\
\text { nido de muestra. }\end{array}$ & $\begin{array}{l}\text { - Mujeres con LES deben recibir asesoramiento preconcepcional y planificación } \\
\text { familiar. } \\
\text { - LES debe estar inactivo } 6 \text { meses antes de concepción. Tras el parto, se } \\
\text { incrementa la actividad del LES. Importancia del control multidisciplinario } \\
\text { para reducir riesgos. } \\
\text { - Complicaciones maternas: cesárea, preeclampsia, hipertensión, aborto } \\
\text { espontáneo, enfermedad tromboembólica e infección posparto. } \\
\text { - Medicamentos seguros: Azatioprina, corticoides e Hidroxicloroquina. Éste } \\
\text { último se recomienda antes y durante el embarazo. Teratogénicos: Metotrexate } \\
\text { y Micofenolato de Mofetilo. Terapia biológica como el Rituximab o el } \\
\text { Belimumab: escasez de estudios sobre teratogenicidad. }\end{array}$ \\
\hline
\end{tabular}




\begin{tabular}{|c|c|c|c|}
\hline $\begin{array}{l}\text { Autor, año y } \\
\text { lugar }\end{array}$ & $\begin{array}{l}\text { Diseño del } \\
\text { estudio }\end{array}$ & Muestra & Principales conclusiones \\
\hline $\begin{array}{l}\text { Moroni G et al. } \\
2016 \text {, Italia }{ }^{16}\end{array}$ & $\begin{array}{l}\text { Revisión } \\
\text { bibliográfica. }\end{array}$ & $\begin{array}{l}\text { Número no } \\
\text { definido de } \\
\text { muestra. }\end{array}$ & $\begin{array}{l}\text { - Mujeres con LES: mortalidad materna } 20 \text { veces mayor por: sepsis, nefritis } \\
\text { lúpica y deterioro renal. LES debe estar inactivo } 6 \text { meses antes de concepción } \\
\text { (sin nefritis ni accidente cerebrovascular). } \\
\text { - Complicaciones fetales: abortos espontáneos, mortinatalidad, muerte neonatal, } \\
\text { parto prematuro y retraso del crecimiento intrauterino. } \\
\text { - Terapia biológica: Rituximab seguro durante el primer trimestre de gestación. } \\
\text { Belimumab: sin registros de teratogenicidad. } \\
\text { - En la actualidad, disminución del } 43 \% \text { de complicaciones fetales con respecto } \\
\text { a los últimos } 40 \text { años. El aumento del número de células TREG es beneficioso } \\
\text { para el embarazo. }\end{array}$ \\
\hline $\begin{array}{l}\text { Barbhaiya M, } \\
2013, \text { USA }^{17}\end{array}$ & $\begin{array}{l}\text { Revisión } \\
\text { bibliográfica. }\end{array}$ & $\begin{array}{l}\text { Número no } \\
\text { definido de } \\
\text { muestra. }\end{array}$ & $\begin{array}{l}\text { - Mujeres con LES: tasa de complicaciones } 2-4 \text { veces mayor. } \\
\text { - } \text { y complicaciones maternas: } \\
\text { - Complicaciones fetales: nacimiento prematuro, retraso del crecimiento } \\
\text { intrauterino y aborto espontáneo. Fetos de madres con anticuerpos positivos: } \\
\text { bloqueo cardíaco congénito. Como prevención: Hidroxicloroquina. } \\
\text { - Medicamentos seguros: corticoides no fluorados, Azatioprina y Ciclosporina. } \\
\text { Pocos datos sobre seguridad de terapia biológica en embarazo (Rituximab o } \\
\text { Belimumab). } \\
\text { - Medicamentos teratogénicos: Micofenolato de Mofetilo y Metotrexate. }\end{array}$ \\
\hline $\begin{array}{l}\text { Lateef A et al. } \\
2017, \text { USA }^{18}\end{array}$ & $\begin{array}{l}\text { Revisión } \\
\text { bibliográfica. }\end{array}$ & $\begin{array}{l}\text { Número no } \\
\text { definido de } \\
\text { muestra. }\end{array}$ & $\begin{array}{l}\text { - La evaluación de autoanticuerpos, actividad de la enfermedad, y el uso de } \\
\text { medicamentos: claves para una óptima planificación. } \\
\text { - Complicaciones maternas y fetales: preeclampsia ( } 16-30 \%) \text {, parto prematuro, } \\
\text { aborto ( } 50 \%) \text {, retraso del crecimiento intrauterino y lupus neonatal (2\%) en } \\
\text { fetos expuestos a anticuerpos anti-Ro y anti-La. } \\
\text { - Tasas más altas de mortalidad materna, trombosis, infección y complicaciones } \\
\text { hematológicas durante el embarazo con LES. } \\
\text { - Importancia de un equipo multidisciplinar. }\end{array}$ \\
\hline $\begin{array}{l}\text { Eudy AM et al. } \\
2017, \text { USA }^{19}\end{array}$ & $\begin{array}{l}\text { Estudio } \\
\text { observacional, } \\
\text { longitudinal } \\
\text { prospectivo. }\end{array}$ & $\begin{array}{l}\text { Pacientes con } \\
\text { LES y artritis } \\
\text { reumatoide } \\
\text { (AR) embara- } \\
\text { zadas. }\end{array}$ & $\begin{array}{l}\text { - En comparación con los embarazos con AR, los embarazos con LES } \\
\text { tuvieron tasas más altas de nacimiento prematuro ( } 24 \% \text { LES vs } 14 \% \text { RA), } \\
\text { preeclampsia ( } 15 \% \text { LES vs } 7 \% \text { RA) y parto por cesárea ( } 48 \% \text { LES vs } 30 \% \\
\text { RA). La mayoría de los nacimientos prematuros entre las mujeres con LES } \\
\text { fueron indicados ( } 70 \% \text { ) por preeclampsia, mientras que los de AR fueron } \\
\text { espontáneos, debido a la ruptura prematura de las membranas. } \\
\text { - De todos los nacimientos, las complicaciones que más se dieron en LES fueron: } \\
\text { prematuridad debido a cesárea por riesgo materno, con medicación indicada } \\
\text { por preeclampsia o hipertensión. }\end{array}$ \\
\hline $\begin{array}{l}\text { Sammaritano } \\
\text { LR et al. 2017, } \\
\text { EEUU } 20\end{array}$ & $\begin{array}{l}\text { Revisión } \\
\text { bibliográfica. }\end{array}$ & $\begin{array}{l}\text { Número no } \\
\text { definido de } \\
\text { muestra. }\end{array}$ & $\begin{array}{l}\text { - Evaluación preconcepcional: valoración de daño de órganos, actividad de la } \\
\text { enfermedad, anticuerpos y medicamentos. Periodo de inactividad de al menos } 6 \\
\text { meses antes de concebir. } \\
\text { Hipertensión ( } 10 \% \text { ) complica el embarazo en el LES. } \\
\text { - Medicamentos seguros: Ácido Acetil Salicílico (Aspirina) a dosis } \\
\text { bajas, Azatioprina, Ciclosporina, Tacrolimus, corticoides no fluorados e } \\
\text { Hidroxicloroquina. } \\
\text { - Medicamentos teratogénicos: AINEs, Micofenolato y Metotrexate (debe } \\
\text { suspenderse } 3 \text { meses antes de concepción). } \\
\text { - Estudios recientes afirman suspender terapia biológica (Rituximab) } 6 \text { meses } \\
\text { antes de la concepción. Otros afirman que no es teratogénico. }\end{array}$ \\
\hline
\end{tabular}




\begin{tabular}{|c|c|c|c|}
\hline $\begin{array}{l}\text { Autor, año y } \\
\text { lugar }\end{array}$ & $\begin{array}{l}\text { Diseño del } \\
\text { estudio }\end{array}$ & Muestra & Principales conclusiones \\
\hline $\begin{array}{l}\text { Bitencourt N, } \\
\text { et al. 2018, } \\
\text { USA }^{21}\end{array}$ & $\begin{array}{l}\text { Revisión } \\
\text { bibliográfica. }\end{array}$ & $\begin{array}{l}\text { Número no } \\
\text { definido de } \\
\text { muestra. }\end{array}$ & $\begin{array}{l}\text { - Mayores complicaciones fetales y maternos del embarazo si: anticuerpos } \\
\text { antifosfolípidos, antecedentes de nefritis lúpica, hipertensión, dosis de } \\
\text { prednisona }>20 \mathrm{mg} / \text { día, trombocitopenia y lupus activo antes y durante el } \\
\text { embarazo. } \\
\text { - Medicamentos seguros: Ácido Acetil Salicílico (Aspirina) a dosis bajas antes } \\
\text { de la } 16 \text { semana de gestación, Hidroxicloroquina, Azatioprina, Tacrolimus, } \\
\text { Belimumab y Rituximab. } \\
\text { - Medicamentos no seguros: corticoides no fluorados, Ciclosporina (riesgo de } \\
\text { parto prematuro y de bajo peso, hipertensión y preeclampsia), Metotrexate y } \\
\text { Micofenolato. } \\
\text { - Inactividad del LES y control de tratamiento } 6 \text { meses antes del embarazo. }\end{array}$ \\
\hline $\begin{array}{l}\text { Cooper WO } \\
\text { et al. } 2014 \text {, } \\
\text { América }^{22}\end{array}$ & $\begin{array}{l}\text { Estudio } \\
\text { observacional } \\
\text { de cohortes. }\end{array}$ & $\begin{array}{l}\text { Mujeres } \\
\text { embarazadas y } \\
\text { con artropatías } \\
\text { inflamatorias, } \\
\text { LES o enferme- } \\
\text { dad inflamato- } \\
\text { ria intestinal. }\end{array}$ & $\begin{array}{l}\text { - La cohorte incluyó } 608 \text { bebés: } 437 \text { con exposición a inmunosupresores durante } \\
\text { el embarazo y } 171 \text { cuyas madres tomaron inmunosupresores antes, pero no } \\
\text { durante el embarazo. } \\
\text { - Resultados: } 25 \text { embarazos }(4,1 \%) \text { con malformaciones congénitas graves y } 10 \\
\text { abortos }(1,6 \%) \text {. Entre los } 113 \text { bebés prematuros con exposiciones durante el } \\
\text { embarazo, } 23(20,4 \%) \text { tuvieron complicaciones neonatales con riesgo vital, y } \\
\text { entre } 485 \text { lactantes a término, } 10(2,1 \%) \text {, también. } \\
\text { - No se observó un aumento significativo de la proporción de malformaciones } \\
\text { congénitas entre los lactantes cuyas madres estuvieron expuestas al } \\
\text { Metotrexato en el primer trimestre. No obstante, este estudio no proporciona } \\
\text { evidencia científica de que el Metotrexato sea seguro para su uso durante el } \\
\text { embarazo. }\end{array}$ \\
\hline $\begin{array}{l}\text { Quinzanos I et } \\
\text { al. 2014, USA }\end{array}$ & $\begin{array}{l}\text { Estudio } \\
\text { observacional } \\
\text { longitudinal } \\
\text { retrospectivo. }\end{array}$ & $\begin{array}{l}122 \text { mujeres } \\
\text { con LES de en- } \\
\text { tre } 18-50 \text { años } \\
\text { y con capacidad } \\
\text { reproductiva. }\end{array}$ & $\begin{array}{l}\text { - Durante este estudio de } 5 \text { años se documentaron } 15 \text { embarazos. } \\
\text { - Resultados: } 65 \text { pacientes ( } 53 \% \text { ) recibieron medicamentos potencialmente } \\
\text { teratogénicos, y } 30(46 \%) \text { tuvieron discusiones documentadas sobre el riesgo } \\
\text { de estos medicamentos. } \\
\text { - El } 50 \% \text { de las pacientes no habían recibido asesoramiento anticonceptivo al } \\
\text { comenzar su tratamiento teratogénico para el LES. }\end{array}$ \\
\hline $\begin{array}{l}\text { Rutan Kartoz } \\
\text { C, 2015, Nueva } \\
\text { Jersey }^{24}\end{array}$ & $\begin{array}{l}\text { Revisión } \\
\text { bibliográfica. }\end{array}$ & $\begin{array}{l}\text { Número no } \\
\text { definido de } \\
\text { muestra. }\end{array}$ & $\begin{array}{l}\text { - Ciclosporina: riesgo de insuficiencia ovárica y pérdida de fertilidad. } \\
\text { - Mujeres con LES: mayor riesgo de infección, eventos trombóticos, parto } \\
\text { prematuro y preeclampsia. Si hay actividad (nefritis), hipertensión pulmonar o } \\
\text { enfermedad cardíaca los riesgos son más graves. } \\
\text { - Medicamentos seguros: Hidroxicloroquina, Aspirina o Heparina en mujeres con } \\
\text { síndrome antifosfolípido, corticoides no fluorados y Azatioprina. } \\
\text { - Medicamentos contraindicados: Belimumab, Ciclofosfamida, Metotrexato y } \\
\text { Micofenolato. } \\
\text { - La incidencia de ansiedad y depresión en embarazadas con LES puede alcanzar } \\
\text { el } 60 \% \text {. }\end{array}$ \\
\hline
\end{tabular}

\section{- Fertilidad}

De modo general, lupus no es sinónimo de infertilidad, sino que más bien se asocia a la edad a la que deciden o pueden ser madres, es decir, a la programación tardía de sus embarazos. Tanto la edad, la afectación de órganos por la enfermedad, y el uso de medicamentos como la Ciclofosfamida, pueden desencadenar una función ovárica anormal o insuficiencia ovárica 8,17,20,21,24. Por lo general, la fertilidad de la mujer con LES no se encuentra afectada si no existe actividad lúpica ${ }^{26}$.

\section{- Complicaciones}

La presencia de numerosas complicaciones maternas y fetales es una de las características más comunes de los embarazos de pacientes con LES. La presencia de enfermedad activa en el momento de la concepción se asocia a un mayor riesgo de brotes durante la gestación, llevando a numerosas complicaciones que ponen en riesgo de forma considerable la vida tanto de la madre como del feto $0^{7-9,13,15-22,24}$. 
La mayoría de los embarazos en mujeres con LES son considerados de alto riesgo (16-30\% comparado con un $5-7 \%$ en mujeres sanas), lo que requieren un control muy estricto que supone: analíticas semanales con recuento de anticuerpos y otros test de laboratorio, evaluación de la función renal (orina de 24 horas) y actividad de la enfermedad, medición de tensión arterial con gran frecuencia, uso de medicamentos por aparición de síntomas de actividad u otro diagnóstico, así como ecografías mensuales a partir de la semana 16 de gestación, pruebas de vigilancia fetal y velocidad Doppler de la arteria umbilical semanales (a partir de la semana 20-24 de gestación), vigilancia suplementaria una vez al mes para posible diagnóstico de crecimiento uterino retardado, etc. ${ }^{7-9,13,15-18,20,24}$.

A pesar de todo el control y seguimiento previamente citado, la clave para un embarazo exitoso está en dos aspectos: que en el momento de la concepción y 6 meses atrás no exista o haya existido actividad del lupus, así como el uso de un tratamiento no teratogénico que cubra las necesidades de la enfermedad $y$, a su vez, intervenga en la no aparición de un brote ${ }^{7-9,13,15-18,20-24}$.

El hecho de que no haya existido actividad del LES en los 6 meses anteriores a la concepción hace que disminuyan considerablemente el número de complicaciones en estos embarazos. Entre esta actividad se encuentran: nefritis lúpica activa o deterioro renal, hipertensión, accidente cerebrovascular en los 6 meses anteriores o casos graves de miocardiopatía, diabetes mellitus, obesidad, enfermedad pulmonar y presencia de autoanticuerpos, entre otras ${ }^{7,9,13,15,16,18,20,21,24}$.

La presencia de LES activo o nefritis previa aumenta significativamente el riesgo de hipertensión y preeclampsia, que se desarrollan en un $14-23 \%$ de los casos y originan partos prematuros y mayor índice de cesáreas ${ }^{8,16-18,24}$. Dicha actividad se mide mediante un control preconcepcional, basado en los riesgos individuales de la paciente en los cuales se incluyen: la historia clínica, pruebas de laboratorio y examen físico; así como el establecimiento de unos objetivos que pretenden lograrse con un seguimiento estrecho de las complicaciones maternas y fetales que puedan aparecer ${ }^{15,20}$.

Las complicaciones maternas están presentes en el 50\% de los casos y se asocian a la actividad del LES. Entre ellas destacan: preeclampsia (14-23\%), eclampsia $(0,5 \%)$, hipertensión, aborto espontáneo $(20 \%)$, parto pretérmino $(21 \%)$, hemorragia preparto y postparto que puede estar ocasionada por cesárea, enfermedad tromboembólica, sepsis, afectación renal o de otros órganos, diabetes gestacional por el uso de glucocorticoides y muerte $^{8,9,13,15,16,18,19,20,24}$.

En cuanto a las morbilidades fetales, dependen de la actividad lúpica que presente la madre antes y durante el embarazo y de la teratogenicidad que suponga el tratamiento que esté tomando. Destacan complicaciones como: malformaciones, prematuridad $(40 \%)$, retraso del crecimiento intrauterino ( $13 \%$ ), lupus neonatal (1$2 \%$ ) por la presencia de anticuerpos anti-La y anti- Ro transferidos pasivamente por el cordón umbilical, bloqueo cardíaco congénito, muerte fetal por aborto espontáneo $(16 \%)$ o muerte neonatal $(2,5 \%)^{7-9,13,15-24}$.

Se ha descubierto que el aumento de las células TREG (células $T$ reguladoras), encargadas del control de las respuestas inmunitarias, hace que disminuya cuantiosamente las complicaciones en los embarazos con LES 7,16 . La estratificación del riesgo se logra evaluando la presencia de anticuerpos, la actividad de la enfermedad, el uso de medicamentos y el control de los síntomas que vayan apareciendo, que deberán ser identificados a tiempo y con la consecuente elaboración de una nueva estrategia de manejo del embarazo ${ }^{18,20,24}$.

Existen otros artículos que corroboran que preeclampsia y eclampsia son las complicaciones maternas que más se dan en pacientes con LES embarazadas, con un 7,6\% y un $0,8 \%$, respectivamente. En ellos se afirma que la presencia de anticuerpos anti-La y anti-Ro está asociado al Lupus Neonatal y al bloqueo cardíaco congénito y que un tratamiento con Hidroxicloroquina parece disminuir el riesgo de padecerlo. Sin embargo, estas complicaciones pueden no aparecer cuando el LES Ileva un largo período inactivo ${ }^{27,28}$.

Recientemente, se ha propuesto que cuatro meses de remisión del LES antes de la concepción son suficientes para asegurar un embarazo exitoso ${ }^{29}$.

Otra complicación importante la cual origina numerosas muertes en pacientes con LES embarazadas es la sepsis. Dado que estas mujeres se encuentran inmunodeprimidas y son susceptibles a contraer infecciones fácilmente, los médicos deben hacer hincapié en el control de síntomas y curación de la infección desde el momento en el que aparece ${ }^{30}$.

\section{- Tratamiento}

A pesar de encontrar contraposiciones con respecto al uso de terapias biológicas como el rituximab o el belimumab, o inmunosupresores como la Ciclosporina 
durante la gestación, existen medicamentos que se consideran teratogénicos y que deberían evitarse absolutamente por el elevado riesgo de causar malformaciones fetales, abortos espontáneos y/o sepsis materna. Entre ellos se encuentran: inmunosupresores como el metotrexate o el micofenolato de mofetilo, los cuales deberán suspenderse o sustituirse por otros al menos 3-6 meses antes de la concepción; AINEs (antiinflamatorios no esteroideos) como el ibuprofeno, ketoprofeno, etc.; glucocorticoides fluorados como la dexametasona o la betametasona; y estrógenos en el caso de padecer también Síndrome Antifosfolípido, por el elevado riesgo de trombosis ${ }^{7-9,15,17,18,20-24}$.

En cambio, el uso de otros medicamentos no teratogénicos puede incluso reducir la incidencia de actividad o brotes en el embarazo, funcionando así como terapia de mantenimiento, y entre ellos destacan: glucocorticoides no fluorados a bajas dosis como la prednisona (no más de $20 \mathrm{mg}$ al día); inmunosupresores como el tacrolimus o la azatioprina, aunque se desconocen los efectos a largo plazo de esta última; el ácido acetil salicílico (aspirina) a dosis bajas, ya que disminuye el riesgo de preeclampsia; y la hidroxicloroquina, medicamento el cual está recomendado antes y durante todo el embarazo por su papel beneficioso en la prevención de brotes y riesgo de bloqueo cardíaco congénito ${ }^{7-9,15-18,20,21,23,24}$.

En contraposición, otros autores aconsejan el uso de corticoides fluorados para reducir la respuesta inflamatoria sobre el corazón del feto y, de este modo, retrasar la evolución del bloqueo cardíaco a bloqueo auriculoventricular completo. Otros, afirman que pueden usarse AINEs de forma puntual a lo largo del embarazo excepto en el último trimestre, por el riesgo del cierre prematuro del ductus arterioso 27,28 .

\section{- Intervenciones de enfermería}

Debido a los grandes problemas que supone un embarazo no controlado en una paciente con LES, es necesario que el equipo multidisciplinar formado por reumatólogos, obstetras, neonatólogos y enfermeras; se encarguen del correcto y minucioso control individualizado de la mujer gestante $8,9,13,15,18,20,21,23,24$.

Enfermería tiene una labor verdaderamente importante en la mujer con LES ya que, en el caso de no encontrarse en el momento idóneo de concebir por su actividad lúpica, deberá usar anticonceptivos y es enfermería quien se encarga de hacer hincapié en la educación sexual, estrategias preventivas y un buen asesoramiento preconcepcional, así como proporcionar apoyo psicoló- gico evitando así el miedo, la falta de información, la ansiedad o la depresión 9,15,18,20,21,23,24.

Algunos autores destacan la importancia del equipo multidisciplinar en el tema del embarazo en mujeres con LES, destacando la evaluación preconceptual de la enfermedad antes de la concepción, es decir, si se encuentra activa o no, así como el reajuste del tratamiento en cuanto a dosis y fármacos permitidos no teratogénicos ${ }^{27}$.

Para reducir la incidencia de complicaciones en el embarazo, especialmente el parto prematuro, y para obtener un buen resultado, es obligatoria una buena preparación antes de la concepción y un buen control de la enfermedad durante toda la gestación. Sin embargo, se necesita evidencia científica sobre la relación entre resultados del embarazo y la actividad lúpica preconcepcional para afirmar lo anteriormente citado ${ }^{26}$.

Nuevos factores inmunológicos, hormonales y ambientales deben ser identificados con el fin de desarrollar estrategias de tratamiento para estas pacientes y reducir las complicaciones del embarazo, tanto maternas como fetales $^{29}$.

\section{Limitaciones del estudio}

La primera limitación ha sido la propia búsqueda en sí, ya que no encontramos abundante bibliografía con evidencia científica acerca del tema, de modo que no pueden establecerse un gran número de hallazgos concluyentes.

Otras limitaciones son, sin duda, que nos topamos con algunas contradicciones con respecto a la medicación utilizada en el LES durante el embarazo, y en el apartado de intervenciones de enfermería, solo encontramos bibliografía basada en recomendaciones en lugar de investigación.

\section{Consideraciones prácticas}

Es fundamental que en pacientes con lupus exista evidencia científica acerca del control de sus embarazos, puesto que se trata de una enfermedad realmente delicada en la que las pacientes se encuentran sometidas bajo un gran número y variedad de medicamentos, muchos de ellos tóxicos y teratógenos y que, antes y durante la gestación, deben suprimir o sustituir por otros para no causar daños en el feto $y$, a su vez, no exacerbar su propia enfermedad. 
Esto hace que aparezcan miedos, ansiedad, depresión u otros trastornos patológicos que hacen que estas mujeres sufran y, muchas de ellas, abandonen la terapia por falsas creencias acerca de su toxicidad.

Para ello, enfermería junto con un equipo multidisciplinar, deben abordar a cada una de estas mujeres de forma integral, aportando en gran medida apoyo psicológico, así como resolviendo sus dudas basándonos en estudios científicos que avalen nuestros objetivos y conseguir que se sientan tranquilas para que su enfermedad no empeore por estrés y sus embarazos se resuelvan con éxito. A las mujeres con LES que deseen quedar embarazadas, para que éstas lo hagan en el momento idóneo que corresponde con la inactividad lúpica A la vista de los resultados de esta revisión, las principales conclusiones que se pueden extraer son que, en la actualidad, la mayoría de las mujeres con LES pueden conseguir su objetivo de la maternidad, siempre y cuando la enfermedad se encuentre controlada al menos 6 meses antes de la concepción y durante todo el embarazo para evitar así complicaciones graves.

Las variables como la fertilidad, las complicaciones o el tratamiento influyen de forma directa sobre la mujer con LES embarazada, ya que deben seguir un control minucioso y exhaustivo de su gestación, el cual será guiado por un equipo multidisciplinar, en el que será importante el papel de Enfermería, colaborando en la planificación familiar y con trol del embarazo.

Recibido: 10-11-19

Revisado: 05-12-19

Modificado: 30-12-19

Aceptado: 15-01-20

\section{Bibliografía}

1. Gómez-Puerta JA, Cervera R. Lupus eritematoso sistémico. Medicina y Laboratorio. 2008;14:21123.

2. Enríquez-Mejía MG. Fisiopatología del lupus eritematoso sistémico. Rev. med. investig. 2013;1(1):8-16.
3. Enciclopedia Médica A.D.A.M. [Internet]. Atlanta (GA): A.D.A.M., Inc.; 2005. Lupus eritematoso sistémico; 2018 ene [Consultado 9 abr 2019]. Disponible en: https://medlineplus.gov/spanish/ency/article/000435.htm.

4. Bãlãnescu $A$, Donisan $T$, Bãlãnescu D. An ever-challenging relationship: lupus and pregnancy. Reumatología. 2017;55(1):29-37.

5. Sánchez-Rodríguez SH, Barajas-Vásquez GE, Ramírez-Alvarado ED, Moreno-García A, Barbosa-Cisneros OY. Lupus eritematoso: enfermedad autoinmune sistémica y órgano específica. Rev Biomed. 2004;15:173-80.

6. Gcelu A. Rheumatic diseases and pregnancy. S Afr Med J. 2014;104(9):643.

7. Tower C, Crocker I, Chirico D, Baker P, Bruce I. SLE and pregnancy: the potential role for regulatory $T$ cell. Nat. Rev. Rheumatol. 2011;7(2):124-8.

8. Soh MC, Nelson-Piercy C. High-risk pregnancy and the rheumatologist. Rheumatology (Oxford). 2015;54(4):572-87.

9. Singh AG, Chowdhary VR. Pregnancy-related issues in women with systemic lupus erythematosus. Int $\mathrm{J}$ Rheum Dis. 2015;18(2):172-81.

10. Abarientos C, Sperber K, Shapiro DL, Aronow WS, Peng Chao C, Ash JY. Hydroxychloroquine in systemic lupus erythematosus and rheumatoid arthritis and its safety in pregnancy. Expert Opin. Drug Saf. 2011;10(5):705-14.

11. Sciascia S, Sciascia S, Hunt BJ, Talavera-García E, Lliso G, Khamashta MA, Cuadrado MJ. The impact of hydroxychloroquine treatment on pregnancy outcome in women with antiphospholipid antibodies. Am J Obstet Gynecol. 2016;214(2):273.el-273. e8.

12. Ponticelli C, Moroni G. Immunosuppression in pregnant women with systemic lupus erythematosus. Expert Rev. Clin. Immunol. 2015;11(5):549-52.

13. Chong Zhang, Mei-ying Liang, Xue Xu, Xue-wu Zhang, Shi Chen. Clinical features of new-onset systemic lupus erythematosus in pregnant patients. J. Obstet. Gynaecol. Res. 2017;44(2):234-40. 
14. Alonso MD, Llorca J, Martínez-Vázquez F, Miranda-Filloy JA, Diaz de Teran T, Dierssen T et al. Systemic Lupus Erythematosus in Northwestern Spain. A 20-Year Epidemiologic Study. Medicine 2011; 90(5):350-8.

15. Andreoli L, Crisafulli F, Tincani A. Pregnancy and reproductive aspects of systemic lupus erythematosus. Curr Opin Rheumatol. 2017;29(5):473-9.

16. Moroni G, Ponticelli C. Pregnancy in women with systemic lupus erythematosus (SLE). Eur J Intern Med. 2016;32:7-12.

17. Barbhaiya M, Bermas BL. Evaluation and management of systemic lupus erythematosus and rheumatoid arthritis during pregnancy. Clin. Inmunol. 2013; 149(2):225-35.

18. Lateef A, Petri M. Systemic Lupus Erythematosus and Pregnancy. Rheum. Dis. Clin. N. Am. 2017; 20(1):87-118.

19. Eudy AM, Jayasundara $M$, Haroun $T$, Neil $L$, James $\mathrm{AH}$, Clowse MEB. Reasons for cesarean and medically indicated deliveries in pregnancies in women with systemic lupus erythematosus. Lupus. 2017; 27(3):351-6.

20. Sammaritano LR. Management of Systemic Lupus Erythematosus During Pregnancy. Annu. Rev. Med. 2017;68:271-85.

21. Bitencourt N, Bermas BL. Pharmacological Approach to Managing Childhood-Onset Systemic Lupus Erythematosus During Conception, Pregnancy and Breastfeeding. Adis. 2018;20(6):511-21.

22. Cooper WO, Cheetham TC, Li DK, Stein CM, CaIlahan ST, Morgan TM et al. Brief report: Risk of adverse fetal outcomes associated with immunosuppressive medications for chronic immune-mediated diseases in pregnancy. Arthritis Rheumatol. 2014;66(2):444-50.
23. Quinzanos I, Davis L, Keniston A, Nash A, Yazdany $\mathrm{J}$, Fransen $\mathrm{R}$ et al. Application and feasibility of systemic lupus erythematosus reproductive health care quality indicators at a public urban rheumatology clinic. Lupus. 2014;24(2):203-9.

24. Rutan Kartoz C. Reproductive health concerns in women with Systemic lupus Erythematosus. MCN. Am J Matern Child Nurs. 2015;40(4):220-6.

25. Guibert Toledano ZM, Reyes Llerena GA, Rigñack Ramírez L, Cruz García Y, Acosta Lapera D, Salgado Galloso SB. Embarazo y puerperio en el lupus eritematoso sistémico. Actualización. Rev. cuba. reumatol. 2013;15(2):71-5.

26. Yang MJ, Chen CY, Chang WH, Tseng JY, Yeh CC. Pregnancy outcome of systemic lupus erythematosus in relation to lupus activity before and during pregnancy. J Chin Med Assoc.2014;78(4):235-40.

27. Rodríguez Almaraz ME, Rabadán Rubio E, Lozano F. Protocolo de manejo del lupus eritematoso sistémico en el embarazo. Medicine. 2017;12(25):1474-77.

28. Ugarte A, Villar I, Ruiz-Irastorza G. Manejo del embarazo en pacientes con lupus eritematoso sistémico. Rev Clin Esp. 2012;212(10):491-8.

29. Jara LJ, Medina G, Cruz-Domínguez P, Navarro C, Vera-Lastra 0, Saavedra MA. Risk factors of systemic lupus erythematosus flares during pregnancy. Inmunol Res. 2014;60(2-3):184-92.

30. Bauer ME, Bateman BT, Bauer ST, Shanks AM, Mhyre JM. Maternal Sepsis Mortality and Morbidity During Hospitalization for Delivery: Temporal Trends and Independent Associations for Severe Sepsis. Anesth Analg. 2013;117(4):944-50.

Este artículo se distribuye bajo una Licencia Creative Commons Atribución-NoComercial 4.0 Internacional. https://creativecommons.org/licenses/by-nc/4.0/

Open Access (C) (1) (8) 